\title{
The Effects of playing the COSMA Cognitive Games in Dementia
}

\author{
Kartheka Bojan ${ }^{1}$, Thanos G. Stavropoulos ${ }^{2}$, Ioulietta Lazarou ${ }^{2,3}$, Spiros \\ Nikolopoulos ${ }^{2}$, Ioannis Kompatsiaris ${ }^{2}$, Magda Tsolaki ${ }^{2,3,4}$, Elizabeta \\ Mukaetova-Ladinska ${ }^{5}$, Aikaterini Christogianni ${ }^{1,6}$ \\ ${ }^{I}$ BrainBerry Ltd., UK, \{kartheka, kate.christogianni\}@brainberry.uk \\ ${ }^{2}$ Information Technologies Institute, Centre for Research and Technology Hellas (CERTH- \\ ITI), Thessaloniki, Greece \\ \{athstavr, iouliettalaz, nikolopo, ikom\}@iti.gr \\ ${ }^{3} 1$ st Department of Neurology, "AHEPA" UGH”, School of Medicine, Faculty of Health \\ Sciences, Aristotle University of Thessaloniki (AUTH), Greece \\ tsolakim1@gmail.com,iouliettalaz@iti.gr \\ ${ }^{4}$ Greek Association of Alzheimer's Disease and Related disorders (GAADRD), Greece \\ tsolakim1@gmail.com \\ ${ }^{5}$ Department of Neuroscience, Psychology and Behaviour, University of Leicester, UK \\ eml12@le.ac.uk \\ ${ }^{6}$ School of Design and Creative Arts, Loughborough University, UK \\ kate.christogianni@brainberry.uk
}

\begin{abstract}
Brain Training games are increasingly gaining attention as a nonpharmacological intervention to promote well-being and quality of life in people living with dementia. Herein we present the COSMA software and a pilot study to evaluate its impact on the emotions of people in the spectrum of dementia. The software was created in accordance to the UK National Institute for Health and Care Excellence (NICE) guidelines as a 'brain-stimulating' software for use by people with cognitive impairment i.e., mild cognitive impairment (MCI) and early dementia.
\end{abstract}

The pilot study aims to investigate whether the current COSMA game designs have an impact on emotions in people with MCI and early dementia. The emotional evaluation before and after playing COSMA games was carried out using the Positive and Negative Affect Schedule (PANAS). Our findings demonstrated a small, but significant increase in positive emotions (MCI: $p=$ 0.041; early dementia: $p=0.042$ ) and decrease in negative emotions (MCI: $p=$ 0.001; early dementia: $p<0.001)$. These preliminary results showed that people with MCI and early dementia experienced positive emotions while playing the COSMA games, suggesting that people with cognitive impairment may benefit from using the COSMA software regularly.

Keywords: Serious games, Dementia, Brain stimulation; 



\section{Introduction}

The ageing population and the increasing number of people in the spectrum, combined with a lack of pharmaceutical treatment, results in an ever-increasing demand for dementia prevention methods. The current non-pharmaceutical interventions include reminiscence therapy, cognitive rehabilitation, occupational therapy, gamification/brain training or neurofeedback therapy focusing on early to moderate dementia patient groups [1].

Brain training apps hold their own unique and noticeable place within the category of nonpharmaceutical dementia therapy. In general, brain training games aim at inspiring creativity [2], improving learning ability [3], psychological well-being [4] and cognitive functions such as attention, language and memory [5], [6], as well as the ability to be self-sufficient [3] and perform daily tasks independently [5], [7].

There are several brain training software on the market, with only a few supported by research studies. Moreover, these research studies have been largely focused on healthy volunteers rather than dementia patients. There is a small number of brain training software designed for people with dementia. Most of the applications are designed for cognitively healthy elders, while other applications have evolved into serious games after a research study was conducted. One such example is the Nintendo Wii-Fit console, which uses physical exercise like yoga, strength training, aerobics and balance games via TV and virtual trainers to assist people with dementia in their environment. Results from this trial have shown a noticeable improvement in balance and gait in people with mild Alzheimer's Disease (AD) in comparison with a walking program which involved the same participants [8]. Other examples include Big Brain Academy (BBA) video games: when compared to the Integrated Psychostimulation Program (IPP), these games showed both slower rates of cognitive decline and significant improvement in depression in AD patients [9].

While the currently available brain training games target the cognitive and physical functions of the users, the social and emotional functions are mostly ignored and, thus, lack definite focus on psychological and behavioural changes. The emotional and/or psychological trauma of early dementia or Mild Cognitive Impairment (MCI) diagnosis can be overwhelming and an occurrence of 'Grief' and 'Guilt' often follows [10]. Similarly, at moderate and severe stages of dementia, patients' apathy, lack of motivation and lack of action are observed and are believed to be an experience of psychological death and loss of individual consciousness [10]-[12]. The expression of these psychological experiences occurs during the early stages and is lost as the disease progresses leading to loss of communication, humiliation, self-doubt, and sense of failure [10], [11]. Focusing, therefore, on the psychological and emotional well-being of people with dementia is as important as cognitive improvement, for both facilitate quality of life and promote longer independent living [13], [14].

We have developed a framework of serious games designed for people with early dementia called 'Cognitive Operational System for Mental Health Advancement' (COSMA). COSMA focuses on stimulating different brain activities like critical thinking, retrieving memories, identifying items, performing tasks, navigational abilities, emotional uplift, etc. The COSMA games are brain-stimulating software referred as 'engagement in a range of activities and discussions that are aimed at general improvement of cognitive and social functioning' by the UK National Institute for Health and Clinical Excellence (NICE) guidelines [15].

COSMA is designed as a therapy tool for people with pre-clinical dementia to moderate stages of dementia. Its flexible and personalised gaming design can be tailored in such a manner that people with severe to later stages of dementia can still use COSMA. It contains 7 game modules (35 games in total) which include reminiscence, visual, daily tasks, language, time-spatial, decision making, and meditation, each focusing on different functionalities of the brain. The cognitive game modules are explained in detail with one game example in each 
module, with the logical and psychological reasons that lead to the development of these modules for early dementia described below.

The COSMA construct contains a website that is activated remotely by family members and/or carers. Family members, caregivers and patients themselves can create an account on the website using their email. Pictures of important personal events and people in the patient's life are being selected and uploaded on the website. The COSMA software application then downloads these pictures and the patient is presented with a set of individualized COSMA games available for him/her to play.

This attribute of the COSMA software promotes the involvement of friends, family and carers in the therapy and allows them to participate remotely. Through sharing memories, pictures and information about life events, the engagement between people with dementia and their carers, friends and family enhances. This might help them meet the emotional and psychological needs of both patients and their families and friends over time, which cannot be met via daily routine online chats or video calls. This is an observation made whilst conducting the present and other ongoing COSMA clinical studies. Many of the volunteers spoke to researchers about the positive memories they retrieved by looking at pictures while uploading them on the COSMA website.

The pictures can also be changed at regular intervals according to patients' needs to avoid boredom or increase the difficulty or freshness of the games. For example, for people in moderate dementia stages, i.e. pictures of family members when young, or recognizable life events can be uploaded to promote emotional well-being. Also, frustrations and confusions with recent pictures or related to events the patients might not remember can be avoided through this selection option. Patients' recent autobiographical memories are more affected than those of past events that are autobiographical and convey emotional value [1].

The Reminiscence module includes 4 games which are personalized to the user and are based on pictures of friends and family and life events where positive memories from the past

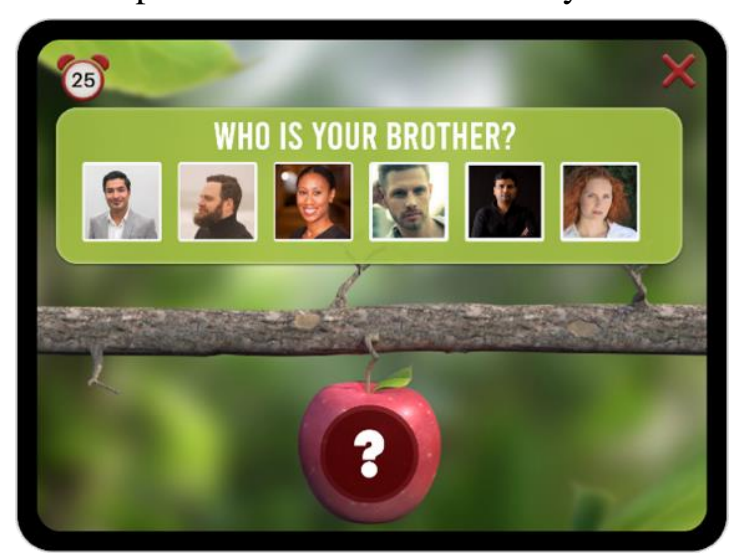

Figure 1. Family Tree game can be retrieved. Reminiscence can help people with dementia by drawing strength from conversations and interactions from long-term memory and provide them with a sense of competence and confidence by using skills they still have [16]. The current state of the art practice of reminiscence includes going through family albums with people with dementia, but COSMA is designed to trigger the most recent memories of events and family members through the fun elements of gaming, since a person with dementia tends to recall better events from the past than from the recent present. For example, the 'Family

tree' game (Figure 1) consists of identifying people in the pictures and helps the participant to remember the relationship he/she has with each of the people depicted. On the top of the screen, a question appears with the request to identify a specific person shown in the pictures. 
The Spatial-Time module contains 5 games in total and aims at triggering both spatial orientation and the concept of space in terms of geographical positions such as the hometown,

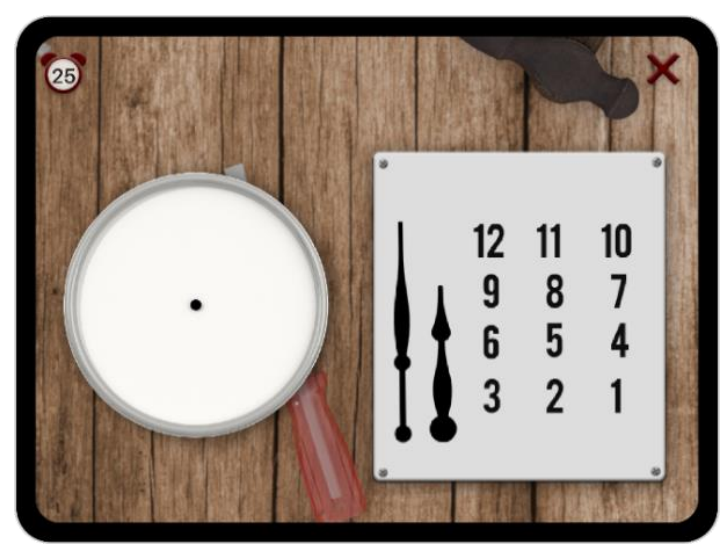

Figure 2. Construct Clock game current living country, time etc. Spatial disorientation is frequently observed in people with dementia, limiting a person's ability to perform daily activities that require navigation independently [17].

The module aims to improve and assess the spatial orientation and time skills in people in the early stages of dementia [17].

The 'Construct clock' game (Figure 2) is an ideal example to explore both spacetime concepts. This game explores the spatial orientation capability of a patient in identifying how they construct the clock, display the numbers, place the clock-hands appropriately and set the instructed time. It is important to note that the clock-drawing test is used for screening cognitive impairment and dementia as a measure of spatial dysfunction and neglect. It is originally used to assess visuo-constructive abilities, but it is also used as a test for dementia diagnosis e.g. Test your Memory (TYM) [18], Clock-Drawing Test [19], General Practitioner Assessment of Cognition Score [20] and Mini-Cog [21] tests, since the test requires verbal understanding, memory and spatially coded knowledge in addition to constructive skills [22].

The construction of the clock game was designed in a therapy form, in which the user follows hints and instructions to construct a clock correctly. The intention is to explore if the continuous gaming practice can trigger the user to identify/read a clock in real-time.

The Language module has 5 games and aims at identifying general or daily used object names for patients to retrieve basic language vocabularies' and identify the objects.

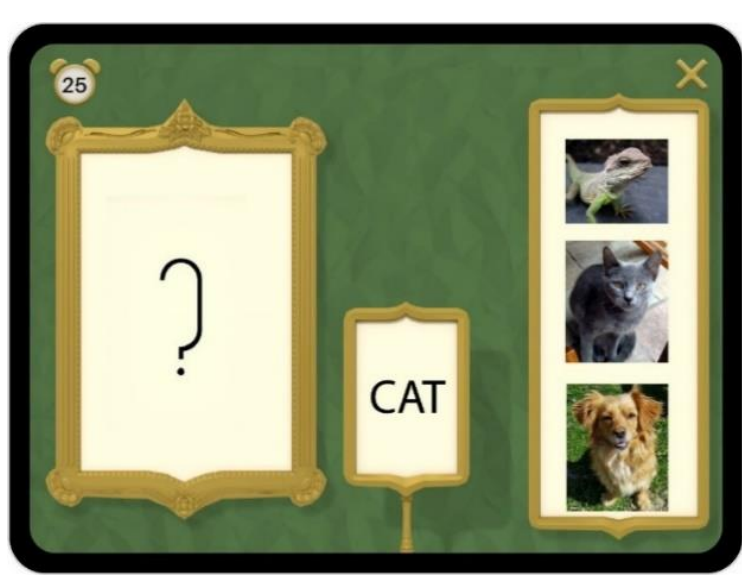

Figure 3. Identify Object Name game Retrieving the name of an object from a pictorial stimulus requires several steps: perceptual analysis of the visual input, access to semantic information on that object, recovery of its lexical label, and, finally, access to its phonological form [23]-[25]. Thus, an apparent effortless task such as naming an item involves many pivotal cognitive processes. The inappropriate functioning of one or several stages will significantly impact patients' naming ability.

For example, the 'Identify Object Name' game (Figure 3) focuses on confrontation naming tasks which have been broadly used to evaluate the cognitive status of neurological patients [26], [27]. The game design uses general and familiar objects for people with memory impairment to refresh their vocabulary. Identification of Objects/items is used also in the screening test for dementia. e.g. Montreal Cognitive Assessment (MoCA) [28] and ACE-III [29]. This COSMA designed module provides digitally many general objects for the patient to recognize, re-register these general objects or simply refresh his/her language and/or object recognition skills [24]. 
The Daily Tasks module is created to train the brain to identify objects used daily and do simple daily tasks activities as per instruction. This module aims to examine whether a long-

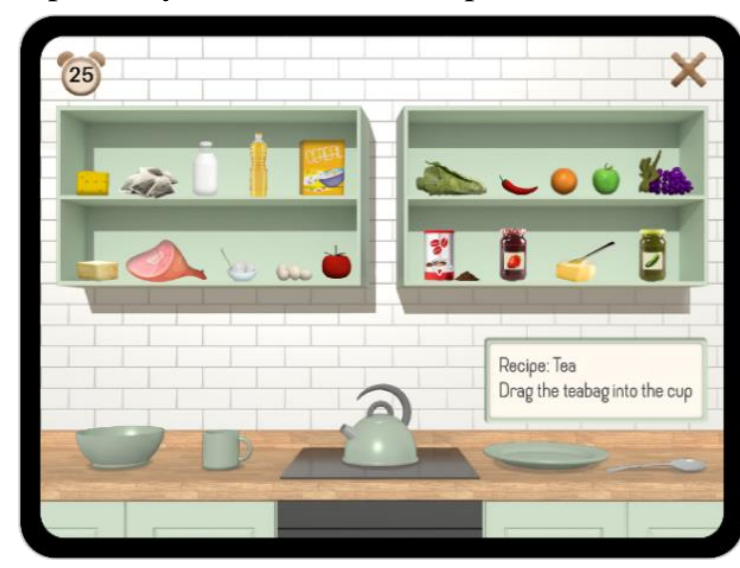

Figure 4. Make a Recipe game time/continuous gaming practice on daily tasks might help people with dementia in real-life to remain functional, i.e. be able to deal with simple daily required routines e.g., making a recipe, gardening etc. The daily task designs are based on European culture and cross-cultural designs will be considered in the future. The decision to use a cooking program as an intervention is based on the Continuity Theory of Ageing. This theory stems from the hypothesis that "in making adaptive choices middle-aged and older adults attempt to preserve and maintain existing psychological and social patterns by applying familiar knowledge, skills, and strategies." [30], [31]. A study on cooking as a therapeutic intervention with 107 participants over three years showed that cooking had a positive effect on behaviour in people living with dementia [32].

The 'Make a Recipe' game (Figure 4) was developed since many people with dementia in residential settings do not have the opportunity to cook or do daily chores. This game encourages the participant to pick ingredients from the kitchen and make a cup of tea or a sandwich as per instructions. The game is designed for the user to identify the items and follow the instructions in a logical order to make a cup of tea which might help him/her to make a cup of tea in real life.

The Decision-Making module introduces 5 games to practice logical thinking when solving a puzzle and improve number skills. Playing number games is not only interesting

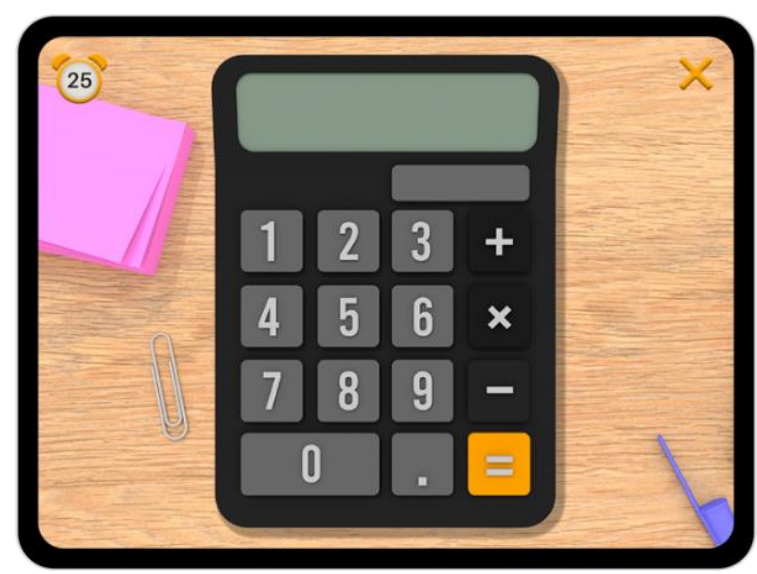

Figure 5. Math Puzzle game and entertaining, it also helps to increase the sense of time, reasoned judgments and distressing/relaxing [33]-[35]. The games focus on logically solving puzzles, recognizing the patterns and deducing solutions to reach the desired one. These puzzles keep the older people engaged since their generation loves solving puzzles; at the same time these games help in sustaining their semantic memory [36]-[38]. For example, the 'Math puzzles' game focuses on developing logical and strategic thinking with number skills. Older people in general enjoy the act of solving sudoku or crossword puzzles. Although to date there is no substantial evidence that these puzzles may improve cognition in people with memory impairment, people report enjoyment when solving puzzles [38], [39]. 
The Visual Tool module deals with visual processing of general shapes, colours and patterns. People with Alzheimer's disease focus more on shapes than colours when

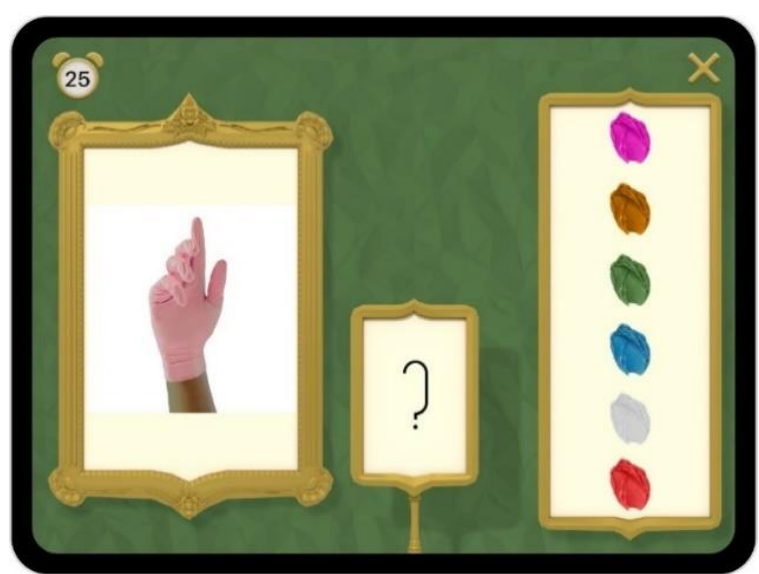

Figure 6. Identify Colours game remembering objects [40]. Remembering an object may surface from combining different attributes and it seems $\mathrm{AD}$ patients lack the ability to 'glue' different kinds of information together. However, studies on the role of colours in object recognition in $\mathrm{AD}$ are controversial. Some studies have shown that patients did not benefit from the addition of colours in the recognition of objects [41], [42], whereas, other studies reported that $\mathrm{AD}$ patients identified objects with colours better, compared to greyscale or black-and-white objects/images [42]-[45].

In the 'Identify colours' game (Figure 6), subjects are instructed to tap and drag the colour that best matches the colour of the user's object/item of daily use in the portrait frame. These games aim to assist with aligning people's familiar objects to colours and shapes; helping them to use different elements of information to identify objects better. The personalized objects might also trigger memories of certain objects that have belonged to the patients /family members, this feeling of familiarity within the game frame can keep the participants engaged with positive emotions.

The Meditation module contains different content like breathing and mindfulness audio files aiming to encourage a habitual process of training the mind. It focuses on personal thoughts and increases the awareness about oneself to promote relaxation and calmness. The meditation scripts are highly focused on positive social behaviour, self-confidence and positive health perspective. People living with early dementia tend to reduce their social life due to their confusion and lack of confidence [46], [47]. There is a plethora of research work carried in the field of meditation interventions which address the overall mental well-being of the human population and aim to tackle anxiety, depression, substance abuse, eating disorders and chronic pain [48]. Mindful meditation is increasingly added in psychotherapeutic programs and many research-based studies are focusing on the benefits of meditation in dementia patients [48]. After a dementia diagnosis, patients tend to hold on to their religion or spiritual beliefs more strongly [48] and this module may also provide spiritual strength to people with dementia [49]-[51]. The audios include: positive hypnosis for selfconfidence and better social interactions, breathing sessions and full-body awareness to boost awareness of the users' positive health condition.

\section{Method}

The study was designed and sponsored by BrainBerry Ltd (BB, 2019) and conducted by the Centre for Research and Technology Hellas (CERTH) following the declaration of Helsinki and is approved by the GAADRD (Greek Association of Alzheimer's Disease and Related Disorders) scientific and ethic committee.

The Study was advertised to people with MCI and early dementia by CERTH at Saint John Day Centre and the Neurological Department of the University Hospital "AHEPA" of Medical School, Aristotle University of Thessaloniki, Greece. The inclusion criteria were: people with a diagnosis of cognitive impairment, 50-80 years of age, being able to read and write, and having computer skills. Participants with visual impairment that required correction with glasses and/or were colour-blind were also eligible to participate. The exclusion criteria enclosed people with memory deficits due to other neurological diseases 
pag. 50

(e.g., multiple sclerosis, Parkinson's disease etc.). Similarly, illnesses like schizophrenia, serious respiratory and cardio/cerebrovascular disease, delirium etc. also lead to exclusion.

Patients with dementia were diagnosed by neuropsychiatrists and neuropsychologists according to their medical and psychological history, the results of their neurological and neuropsychological assessments as well as structural magnetic resonance imaging (MRI) examination [52]. They fulfilled the Diagnostic and Statistical Manual of Mental Disorders (DSM-5) criteria for dementia of Alzheimer type [53] and the National Institute of Neurological and Communication Disorders and Stroke/Alzheimer's Disease and Related Disorders Association (NINCDS-ADRDA) criteria for probable Alzheimer's Disease (AD) and Vascular Dementia (VD) [54]. Patients with MCI were diagnosed according to the criteria by Petersen et al. [55], [56]. The Dementia Severity Rating Scale (DSRS) [56] was also used to select the participants needed for the study.

Thirty participants were included in the study and played the COSMA games: 15 people with MCI [2 Males (mean age \pm standard deviation): 71.5 years $\pm 0.71 ; 13$ Females: 69.84 years \pm 7.79 ] and 15 people with Alzheimer's disease or Vascular dementia (mild AD, moderate AD, mild VD, moderate VD): 8 Males: 77.63 years $\pm 4.41 ; 7$ Females: 77.42 years \pm 2.94 . The average DSRS score in the MCI group was 7.46 (min: 3, max: 11), and 17.93 (min: 12, max: 23) for the dementia group.

To evaluate the emotional response, the Positive and Negative Affect Schedule (PANAS) questionnaire, a self-reported questionnaire with two 10-item scales to measure both positive and negative emotions [58] was used. The self-reported positive/negative emotions are rated on a 5-point scale (1=not at all, 5=very much) for each item [58]. The higher the number, the higher the Positive or the Negative Affect; the lower the number, the lower the Positive or the Negative Affect.

The PANAS scale has been widely used in identifying altered emotions in people with MCI and dementia, their attitude towards therapeutic and rehabilitation interventions [59] and also for assessing the association of positive and negative feelings i.e. self-esteem, life satisfaction, stress, sleep disruptions [60] in their daily life activities. The scale has been shown to have a satisfactory internal consistency, reliability, test-retest reliability and convergent and discriminant validity in both clinical and non-clinical populations [58], [61].

\subsection{Sample size calculations}

No sample size calculation was made for the pilot study and rule of thumb of minimum 30 participants was considered for the study. According to previous research the "samples with N's between 10 and 30 have many practical advantages" in behavioural pilot research since data are easy to calculate and their analysis is easy to implement [62], [63].

\subsection{Study design and procedure}

During the study day, the interested participants arrived at the laboratory facilities and filled out informed consent forms and DSRS to determine the eligibility criteria and the group they belonged to in the study i.e., MCI or dementia. Six people with AD were accompanied by their caregivers during the testing time.

Once the participant was selected, (s)he was asked to fill out the PANAS questionnaire [58] in a relaxed state to measure his baseline emotional status on that particular day. The participants were asked to bring their family and memory pictures, which were uploaded in the COSMA website with the assistance of the researchers. The researchers guided the participants on how to play the COSMA games. All participants unanimously chose a tablet to play COSMA games and ignored the PC/desktop computer option provided. Participants played the COSMA games independently for approximately 1 hour and 30 minutes. Assistance was provided to two participants with early dementia during their COSMA playing session. 
All the participants were presented with the following 15 games out of the 35 total games from COSMA (at least 2 games from each module): Family Tree, Match the Events, Jigsaw Puzzle, Identify Shapes, Identify Colors, Math Puzzles, Make a Recipe, Calendar, Grow your Garden, Spot The Area, Construct the Time, Construct your Clock, Identify Objects 1, Identify Objects 2, Identify Objects 3. After participants played the games, the PANAS questionnaire was administered again to investigate the positive and negative emotional impact on the target groups. The study was completed in one visit which approximately took 2 hours 30 minutes for each participant.

\subsection{Statistical analysis}

The PANAS scores obtained prior and post-play of COSMA games were analyzed using the SPSS v.25. The t-Paired tests were performed to determine the efficacy of the psychological measurement between the two target groups studied. The descriptive statistics and the t-tests were presented as means $\pm \mathrm{SD}$ (standard deviation), 95\% CI, and significance level ( 2 tailed) at $\alpha=0.05$.

\section{Results}

Both groups showed an increase in their Positive Affect after playing the COSMA games (MCI: pre mean 31.07 \pm 4.64 vs post mean 32.6 \pm 3.76 ; $\mathrm{t}(14)=2.25$, $\mathrm{p}=0.041$, CI: 95\% [3.00, 0.07]; Dementia: pre mean $29.07 \pm 6.17$ vs post mean $31.2 \pm 4.95 ; \mathrm{t}(14)=2.23, \mathrm{p}=0.042$, CI: 95\% [4.18, 0.08] (Figure 7).

The Negative Affect in the two groups decreased after playing the games (MCI: pre mean $19.5 \pm 3.81$ vs post mean $17.2 \pm 2.18 ; \mathrm{t}(14)=-3.95, \mathrm{p}=0.001, \mathrm{CI}$ : $95 \%[-1.04,-3.50]$ Dementia: pre mean $22 \pm 5.88$ vs post mean $18.33 \pm 3.02) ; \mathrm{t}(14)=-4.15, \mathrm{p}<0.001, \mathrm{CI}$ : $95 \%[-1.78,-5.56]$ (Figure 7).

\section{Average PANAS values for $\mathrm{MCl}$ and Dementia pre and post playing COSMA}

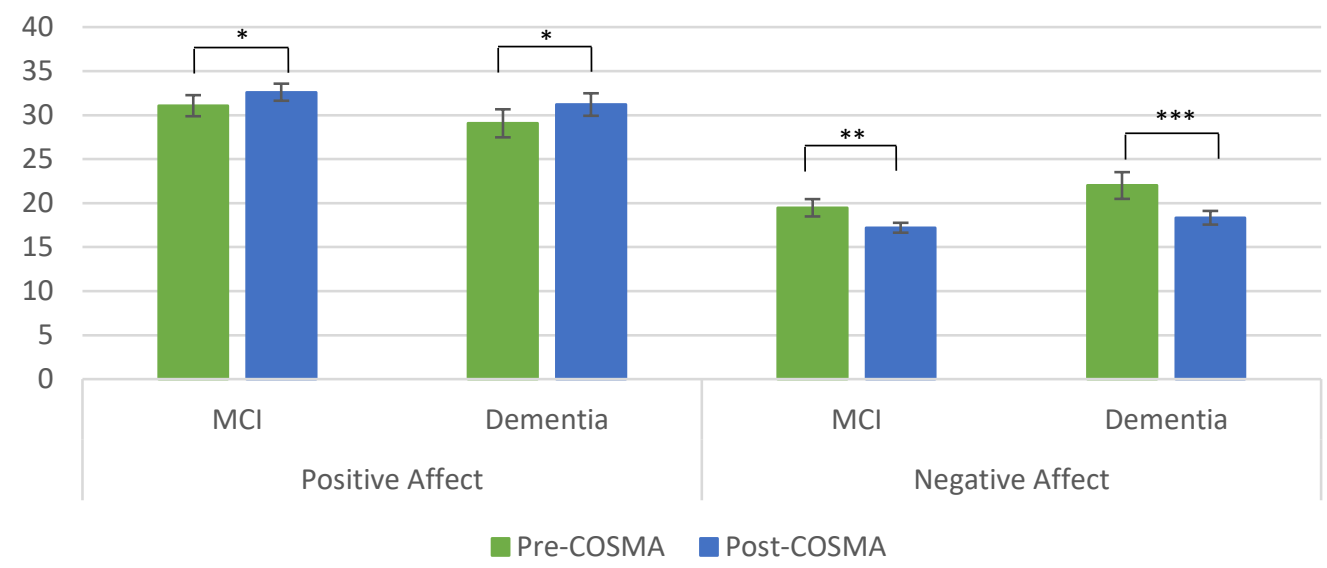

. Figure 7. Emotional impact of COSMA games as assessed with PANAS scale. ${ }^{*}=\mathrm{p} \leq 0.05$; $* *=\mathrm{p} \leq 0.01 ; * * *=\mathrm{p} \leq 0.001$. 
The positive and negative affect variables which showed statistical significance after playing the COSMA games were: Interested, Excited, Alert, and Inspired (positive affect) and Guilty, Distressed, Scared, Irritable, Jittery and Afraid (negative affect) (Table 1, Figure $8)$.

Table 1. Positive and Negative Affect words in pre- and post-playing of COSMA games in MCI and Dementia groups.

\begin{tabular}{|c|c|c|c|c|c|c|}
\hline & & \multicolumn{2}{|l|}{ MCI } & & \multicolumn{2}{|c|}{ Dementia } \\
\hline & & $t(14)$ & $p$ values & & $t(14)$ & $p$ values \\
\hline \multirow{10}{*}{ 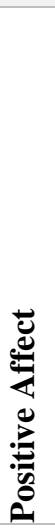 } & Interested & -2.65 & 0.019 & Interested & -3.15 & 0.007 \\
\hline & Excited & -1.38 & 0.189 & Excited & -2.82 & 0.014 \\
\hline & Strong & 0.81 & 0.433 & Strong & 1.87 & 0.082 \\
\hline & Enthusiastic & -1 & 0.334 & Enthusiastic & -1.7 & 0.111 \\
\hline & Proud & -1.78 & 0.096 & Proud & -0.69 & 0.499 \\
\hline & Alert & -2.26 & 0.041 & Alert & -1.87 & 0.082 \\
\hline & Inspired & -1.38 & 0.189 & Inspired & -2.65 & 0.019 \\
\hline & Determined & -0.56 & 0.582 & Determined & -0.69 & 0.499 \\
\hline & Attentive & 0.56 & 0.582 & Attentive & -1.78 & 0.096 \\
\hline & Active & -1.38 & 0.189 & Active & 1.29 & 0.217 \\
\hline \multirow{10}{*}{ 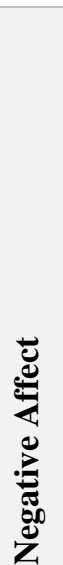 } & Distressed & 1.87 & 0.082 & Distressed & 3.56 & 0.003 \\
\hline & Upset & 1.47 & 0.164 & Upset & 1.15 & 0.271 \\
\hline & Guilty & 2.65 & 0.019 & Guilty & 1.58 & 0.136 \\
\hline & Scared & 1.38 & 0.189 & Scared & 2.26 & 0.041 \\
\hline & Hostile & 1.74 & 0.104 & Hostile & 1.47 & 0.164 \\
\hline & Irritable & 1.38 & 0.189 & Irritable & 2.82 & 0.014 \\
\hline & Ashamed & 1.47 & 0.164 & Ashamed & 1.87 & 0.082 \\
\hline & Nervous & 1.74 & 0.104 & Nervous & 1.82 & 0.089 \\
\hline & Jittery & 2.09 & 0.055 & Jittery & 2.26 & 0.041 \\
\hline & Afraid & 1.87 & 0.082 & Afraid & 3.15 & 0.007 \\
\hline
\end{tabular}

The percentage (\%) change in MCI's before and after COSMA play was $+6.80 \%$ for Interested, $+5.40 \%$ for Alert and Guilt by $-6.80 \%$ (Fig 2). The dementia group exhibited $+12 \%$ for Interested, $+9.20 \%$ for Excitement, $+6.60 \%$ for Inspired, $-14.60 \%$ for Distressed, Scared by $-5.20 \%$, Irritable by $-9.40 \%$, Jittery by $-5.40 \%$ and Afraid by $-12 \%$. 
Positive Affect MCI

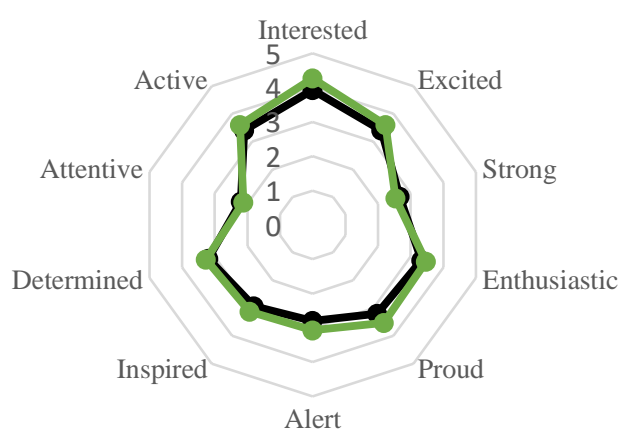

Negative Affect MCI

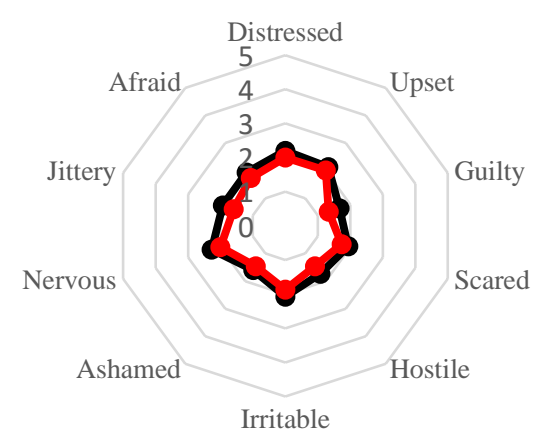

Positive Affect Dementia

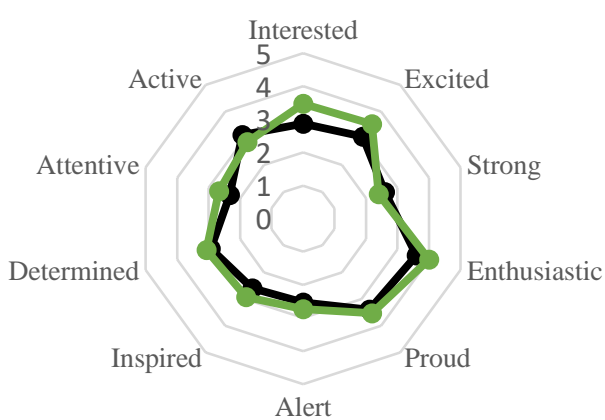

Negative Affect Dementia

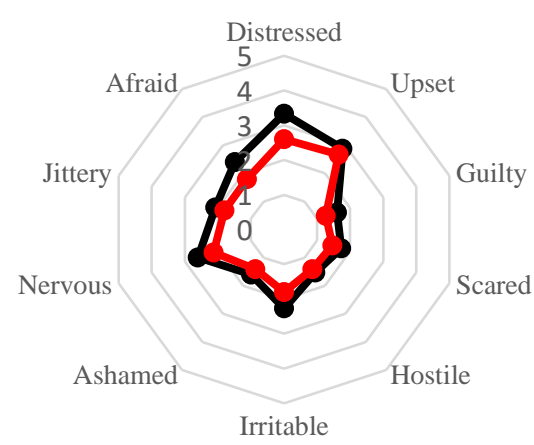

Figure 8. The pre and post-PANAS measurements. The pre and post-PANAS measurements are presented for both MCI and dementia group in black colour for the measurements before playing COSMA; green colour (positive affect) and red colour (negative affect) for the questionnaire measurements after playing COSMA.

\section{Discussion}

This study investigated whether playing the COSMA games influence any emotional changes in people with either early dementia or MCI. Our results indicate that the COSMA games trigger specific positive responses whilst decreasing certain negative responses in both groups, MCI and dementia.

The PANAS questionnaire is designed to assess the affect in several contexts, with scores showing indicating variations in one's emotions [58]. We consider high positive affect scores to indicate pleasurable engagement, whereas low positive affect scores to show the opposite [58]. The study showed that there was a decrease in 'Guilt' in the MCI group, which was considered a noticeable emotional change, that could have been triggered from the achievement of completion of the COSMA games. We have to stress that the assessment was based on the self-reported emotional status of the person at the time of the activity (i.e. playing the games) and it remains unknown whether these emotional states are sustained for longer.

It is interesting to note that only the negative feeling of 'Guilt' was reduced in the MCI participants, whilst in the dementia group the feelings of 'Irritable', 'Distress', 'Afraid', 'Scared' and 'Jittery' showed a decrease. Since people with dementia experience entwined emotions of fear and anxiety as their disease progresses, witnessing these negative emotions 
pag. 54

being subdued after playing COSMA suggests a possible alternative way of regulating the emotional burden.

The decrease in these specific negative emotions might have occurred due to the games having (i) family and friends-based pleasant memory pictures and daily activities-based games inducing familiarity (distress and scared); (ii) fun, colourful, appropriate gaming level and user-friendly gaming designs (jittery and irritable) and (iii) the logic of the game designs being relatable and individualized to achieve cognitive achievement or rewards in the games (afraid). An additional explanation for this observation could also be patients' interactions with researchers while uploading family pictures. This exchange could have also contributed towards reducing their psychological worries and anxiety.

In terms of positive emotions, both groups showed an increased feeling of 'Interest' which indicates their enjoyable engagement with the COSMA games during the session. Apart from 'Interest', the MCI group showed 'Alert', whereas in the dementia group 'Excitement' and 'Inspiration' indicated a positive emotional change.

The observation of the feeling of 'Alert' and 'Interest' in patients with cognitive decline is essential to complete daily tasks and improve attention [64], [65]. Our study, as supported via the increase in positive emotional feelings, shows that COSMA games are enjoyable and well received by participants.

\section{Limitations and future studies}

Our study has several limitations, starting from the small number of participants to the short study duration and the absence of a follow-up to indicate the lasting effects of the emotional observations made. Participants might have also reported a positive emotional state to promote social desirability [66], out of fear of not being accepted and liked by their peers, leading to a bias in the study results. Our researchers focused on a friendly approach and encountered all participants with warmth, and this may have played a role in generating positive emotions. Also, participants' emotional changes could be related to the novelty effect, i.e., an improvement in performance when using new technologies. We have yet to investigate the emotional impact of COSMA when used for a longer time. This is important to consider since people with dementia and MCI have difficulties engaging in tasks due to apathy and lack of interest [67]. Future studies may need to be based on a more comprehensive neuropsychological battery to assess if the COSMA games have an impact on depression, stress, anxiety, apathy and motivation in patients with MCI and dementia, and take into account the prolonged usage of COSMA games and measure its long-term effect, with a follow-up study.

\section{Conclusion}

We demonstrate an overall positive emotional change in both MCI and dementia people playing COSMA games. The different emotional status remarked in both groups emphasizes the different psychological aspects associated with the different stages of cognitive impairment. Our study also demonstrates that participants experienced positive emotions by playing COSMA games.

\section{Funding information}

The study is funded by BrainBerry Ltd. in the UK. 


\section{References}

[1] C. E. Hou, B. L. Miller, and J. H. Kramer, "Patterns of autobiographical memory loss in dementia," Int. J. Geriatr. Psychiatry, vol. 20, no. 9, pp. 809-815, Sep. 2005, doi: $10.1002 /$ gps. 1361.

[2] A. Sisarica, N. Maiden, D. Morosini, L. Panesse, K. Pudney, and M. Rose, "Creativity support in a serious game for dementia care," in Proceedings of the 9th ACM Conference on Creativity \& Cognition, Sydney, Australia, 2013, pp. 349-352, doi:

10.1145/2466627.2466668.

[3] C. Tziraki, R. Berenbaum, D. Gross, J. Abikhzer, and B. M. Ben-David, "Designing Serious Computer Games for People With Moderate and Advanced Dementia: Interdisciplinary Theory-Driven Pilot Study," JMIR Serious Games, vol. 5, no. 3, Jul. 2017, doi: 10.2196/games.6514.

[4] J. Cha et al., "Finding critical features for predicting quality of life in tablet-based serious games for dementia," Qual. User Exp., vol. 4, no. 6, Dec. 2019, doi: 10.1007/s41233-019$0028-2$.

[5] L. Tárraga et al., "A randomised pilot study to assess the efficacy of an interactive, multimedia tool of cognitive stimulation in Alzheimer's disease," J. Neurol. Neurosurg. Psychiatry, vol. 77, no. 10, pp. 1116-1121, Oct. 2006, doi: 10.1136/jnnp.2005.086074.

[6] G. J. Lee et al., "A comparison of the effects between 2 computerized cognitive training programs, Bettercog and COMCOG, on elderly patients with MCI and mild dementia A single-blind randomized controlled study," Med. (United States), vol. 97, no. 45, Nov. 2018 , doi: 10.1097/MD.0000000000013007.

[7] S. McCallum, "Gamification and serious games for personalized health," Stud Health Technol Inform, vol. 177, no. February, pp. 85-96, doi: 10.3233/978-1-61499-069-7-85.

[8] K. P. Padala et al., "Wii-fit for improving gait and balance in an assisted living facility: A pilot study," J. Aging Res., Jun. 2012, doi: 10.1155/2012/597573.

[9] B. Fernández-Calvo, R. Rodríguez-Pérez, I. Contador, A. Rubio-Santorum, and F. Ramos, "Eficacia del entrenamiento cognitivo basado en nuevas tecnologías en pacientes con demencia tipo Alzheimer," Psicothema (Oviedo), vol. 23, no. 1, pp. 44-50, Feb. 2011.

[10] K. J. Doka, "Grief, multiple loss and dementia," Bereave. Care, vol. 29, no. 3, pp. 15-20, Dec. 2010, doi: 10.1080/02682621.2010.522374.

[11] D. E. Clarke et al., "Apathy in Dementia: Clinical and Sociodemographic Correlates," J. Neuropsychiatr., vol. 20, no. 3, pp. 337-347, Jul. 2008, doi: 10.1176/appi.neuropsych.20.3.337.

[12] T. Kitwood, "The experience of dementia," Aging Ment. Heal., vol. 1, no. 1, pp. 13-22, Jun. 1997, doi: 10.1080/13607869757344.

[13] L. D. Oliver et al., "Parsing cognitive and emotional empathy deficits for negative and positive stimuli in frontotemporal dementia," Neuropsychologia, vol. 67, pp. 14-26, Jan. 2015, doi: 10.1016/j.neuropsychologia.2014.11.022.

[14] J. Jolles, "Chapter 2 Cognitive, emotional and behavioral dysfunctions in aging and dementia," in Aging of the Brain and Alzheimer's Disease, vol. 70, D. F. Swaab, E. Fliers, M. Mirmiran, W. A. Van Gool, and F. Van Haaren, Eds. Elsevier, 1986, pp. 15-39.

[15] M. Orrell et al., "Support at Home: Interventions to Enhance Life in Dementia (SHIELD) evidence, development and evaluation of complex interventions," Program. Grants Appl. Res., vol. 5, no. 5, 2017, doi: 10.3310/pgfar05050.

[16] SCIE, "Reminiscence for people with dementia." [Online]. Available: https://www.scie.org.uk/dementia/living-with-dementia/keeping-active/reminiscence.asp. [Accessed: 08-Aug-2020].

[17] L. Liu, L. Gauthier, and S. Gauthier, "Spatial disorientation in persons with early senile dementia of the Alzheimer type," Am. J. Occup. Ther., vol. 45, no. 1, pp. 67-74, Jan. 1991, doi: 10.5014/ajot.45.1.67.

[18] P. Hancock and A. J. Larner, "Test Your Memory test: diagnostic utility in a memory clinic population," Int. J. Geriatr. Psychiatry, vol. 26, no. 9, pp. 976-980, 2011, doi: 10.1002/gps.2639.

[19] I. Aprahamian, J. E. Martinelli, A. L. Neri, and M. S. Yassuda, "The Clock Drawing Test A review of its accuracy in screening for dementia," Dement. Neuropsychol., vol. 3, no. 2, June, 2009, doi: 10.1590/S1980-57642009DN30200002. 
[20] H. Brodaty et al., "The GPCOG: A new screening test for dementia designed for general practice," J. Am. Geriatr. Soc., vol. 50, no. 3, 2002, doi: /10.1046/j.1532-5415.2002.50122.x.

[21] S. Borson, J. M. Scanlan, P. Chen, and M. Ganguli, "The Mini-Cog as a screen for dementia: Validation in a population-based sample," J. Am. Geriatr. Soc., vol. 51, no. 10, 2003, doi: 10.1046/j.1532-5415.2003.51465.x

[22] B. Agrell and O. Dehlin, "The clock-drawing test," Age Ageing, vol. 27, no. 3, pp. 399-403, May 1998, doi: 10.1093/ageing/27.3.399.

[23] G. W. Humphreys, M. J. Riddoch, and P. T. Quinlan, "Cascade processes in picture identification," Cogn. Neuropsychol., vol. 5, no. 1, pp. 67-104, Aug. 1988, doi: $10.1080 / 02643298808252927$.

[24] M. J. Riddoch and G. W. Humphreys, "A case of integrative visual agnosia,” Brain, vol. 110, no. 6, pp. 1431-1462, Dec. 1987, doi: 10.1093/brain/110.6.1431.

[25] M. J. Riddoch and G. W. Humphreys, "Picture naming.," in Visual object processing: A cognitive neuropsychological approach., Hillsdale, NJ, US: Lawrence Erlbaum Associates, Inc, 1987, pp. 107-143.

[26] K. A. Bayles and C. K. Tomoeda, "Confrontation naming impairment in dementia," Brain Lang., vol. 19, no. 1, pp. 98-114, May 1983, doi: 10.1016/0093-934X(83)90057-3.

[27] F. J. Moreno Martínez, "A review of the main tasks used to assess the semantic impairment in Alzheimer's Disease," Acción Psicológica, vol. 4, no. 1, pp. 57-68, 2012, doi: 10.5944/ap.4.1.493.

[28] T. Smith, N. Gildeh, and C. Holmes, "The Montreal cognitive assessment: Validity and utility in a memory clinic setting," Can. J. Psychiatry, vol. 52, no. 5, pp. 329-332, May 2007 , doi: $10.1177 / 070674370705200508$.

[29] P. S. Mathuranath, P. J. Nestor, G. E. Berrios, W. Rakowicz, and J. R. Hodges, "A brief cognitive test battery to differentiate Alzheimer's disease and frontotemporal dementia," Neurology, vol. 55, no. 11, pp. 1613-1620, Dec. 2000, doi:

10.1212/01.wnl.0000434309.85312.19

[30] R. C. Atchley, "Continuity theory and the evolution of activity in later adulthood.," in Activity and aging: Staying involved in later life., Thousand Oaks, CA, US: Sage Publications, Inc, 1993, pp. 5-16.

[31] J. Diggs, "The Continuity Theory of Aging," in Encyclopedia of Aging and Public Health, S. J. D. Loue and M. Sajatovic, Eds. Boston, MA: Springer US, 2008, pp. 233-235.

[32] S. Fitzsimmons and L. L. Buettner, "A therapeutic cooking program for older adults with dementia: Effects on agitation and apathy," Am. J. Recreat. Ther., vol. 2, no. 4, pp. 23-33, 2003.

[33] B. A. Lilliston, D. Brush, and F. H. McDowell, "Day Care for Dementia Patients: An Analysis of a Four-year Program,” J. Am. Geriatr. Soc., vol. 32, no. 12, Dec.1984, doi: 10.1111/j.1532-5415.1984.tb00887.x.

[34] G. F. He, J. W. Park, S. K. Kang, and S. T. Jung, "Development of gesture recognition-based serious games," in Proceedings - IEEE-EMBS International Conference on Biomedical and Health Informatics: Global Grand Challenge of Health Informatics, BHI 2012, 2012, pp. 922-925.

[35] H. Chi, E. Agama, and Z. G. Prodanoff, "Developing serious games to promote cognitive abilities for the elderly," in 2017 IEEE 5th International Conference on Serious Games and Applications for Health, SeGAH 2017, Perth, WA, 2017, pp. 1-8.

[36] M. A. Kraut, S. Kremen, L. R. Moo, J. B. Segal, V. Calhoun, and J. Hart, “Object activation in semantic memory from visual multimodal feature input," J. Cogn. Neurosci., vol. 14, no. 1, pp. 37-47, Jan. 2002, doi: 10.1162/089892902317205302.

[37] A. Astell, "Technology and fun for a happy old age," in Technologies for active aging, Sixsmith A., Gutman G., Ed., Springer, vol. 9, 2013, ch. 10, pp. 169-187.

[38] M. Truscott, "Healthy, Happy, and Hopeful: Complimentary Care for Dementia," Alzheimers. care today, vol. 7, no. 1, pp. 3-6, Jan. 2006.

[39] R. G. Logsdon, S. M. McCurry, and L. Teri, "Evidence-based interventions to improve quality of life for individuals with dementia," Alzheimers. care today, vol. 8, no. 4, p. 309, 2007.

[40] N. S. Foldi et al., "Effects of Graded Levels of Physical Similarity and Density on Visual Selective Attention in Patients With Alzheimer's Disease.," Neuropsychology, vol. 19, no. 1, pp. 5-17, Jan. 2005, doi: 10.1037/0894-4105.19.1.5.

[41] R. L. Adlington, K. R. Laws, and T. M. Gale, "Visual processing in Alzheimer's disease: Surface detail and colour fail to aid object identification," Neuropsychologia, vol. 47, no. 12, 
pp. 2574-2583, Oct. 2009, doi: 10.1016/j.neuropsychologia.2009.05.004.

[42] F. J. Moreno-Martínez and I. C. Rodríguez-Rojo, "On colour, category effects, and Alzheimer's disease: A critical review of studies and further longitudinal evidence," Behav. Neurol., vol. 2015, May 2015, doi: 10.1155/2015/960725.

[43] P. Montanes, M. C. Goldblum, and F. Boller, "The naming impairment of living and nonliving items in Alzheimer's disease," J. Int. Neuropsychol. Soc., vol. 1, no. 1, pp. 39-48, Feb. 1995, doi: 10.1017/S1355617700000084.

[44] H. Chainay and V. Rosenthal, "Naming and picture recognition in probable Alzheimer's disease: effects of color, generic category, familiarity, visual complexity and shape similarity.," Brain Cogn., vol. 1, Nov. 1996.

[45] G. D. Zannino, R. Perri, C. Caltagirone, and G. A. Carlesimo, "Category-specific naming deficit in Alzheimer's disease: The effect of a display by domain interaction," Neuropsychologia, vol. 45, no. 8, pp. 1832-1839, Apr. 2007, doi: 10.1016/j.neuropsychologia.2006.12.011.

[46] S. Arlt, R. Lindner, A. Rösler, and W. von Renteln-Kruse, "Adherence to medication in patients with dementia," Drugs Aging, vol. 25, no. 12, pp. 1033-1047, 2008, doi: 10.2165/0002512-200825120-00005.

[47] L. Frank et al., "Impact of cognitive impairment on mild dementia patients and mild cognitive impairment patients and their informants," Int. Psychogeriatrics, vol. 18, no. 1, p. 151, Jan. 2006, doi: 10.1017/S1041610205002450.

[48] B. K. Hölzel et al., "Mindfulness practice leads to increases in regional brain gray matter density," Psychiatry Res. - Neuroimaging, vol. 191, no. 1, pp. 36-43, Jan. 2011, doi: 10.1016/j.pscychresns.2010.08.006.

[49] J. Bute and L. Morse, Dementia from the Inside: A doctor's personal journey of hope. SPCK, 2018.

[50] J. Russell-Williams, W. Jaroudi, T. Perich, S. Hoscheidt, M. El Haj, and A. A. Moustafa, "Mindfulness and meditation: Treating cognitive impairment and reducing stress in dementia," Rev. Neurosci., vol. 29, no. 7, pp. 791-804, Feb. 2018, doi: 10.1515/revneuro2017-0066.

[51] E. B. Ryan, L. S. Martin, and A. Beaman, "Communication strategies to promote spiritual well-being among people with dementia.," J. Pastoral Care Counsel., vol. 59, no. 1-2, pp. 43-55, Mar. 2005, doi: 10.1177/154230500505900105.

[52] R. C. Petersen, "How early can we diagnose Alzheimer disease (and is it sufficient)? The 2017 Wartenberg lecture," Neurology, vol. 91, no. 9, pp. 395-402, Aug. 2018, doi: 10.1212/WNL.0000000000006088.

[53] American Psychiatric Association, American Psychiatric Association, 2013. Diagnostic and statistical manual of mental disorders (5th ed.). 2013.

[54] G. McKhann, D. Drachman, M. Folstein, R. Katzman, D. Price, and E. M. Stadlan,“Clinical diagnosis of Alzheimer's disease: report of the NINCDS-ADRDA Work Group under the auspices of Department of Health and Human Services Task Force on Alzheimer's Disease.," Neurology, vol. 34, no. 7, pp. 939-944, Jul. 1984, doi: 10.1212/WNL.34.7.939.

[55] R. C. Petersen, G. E. Smith, S. C. Waring, R. J. Ivnik, E. Kokmen, and E. G. Tangelos, “Aging, Memory, and Mild Cognitive Impairment," Int. Psychogeriatrics, vol. 9, no. 1, pp. 65-69, Jan. 1997, doi: 10.1017/S1041610297004717.

[56] R. C. Petersen et al., "Mild Cognitive Impairment: Ten Years Later," Arch. Neurol., vol. 66, no. 12, pp. 1447-1455, Dec. 2009, doi: 10.1001/archneurol.2009.266.

[57] C. M. Clark and D. C. Ewbank, "Performance of the dementia severity rating scale: A caregiver questionnaire for rating severity in Alzheimer disease," Alzheimer Dis. Assoc. Disord., vol. 10, no. 1, pp. 31-39, Apr. 1996, doi: 10.1097/00002093-199601010-00006.

[58] D. Watson, L. A. Clark, and A. Tellegen, "Development and validation of brief measures of positive and negative affect: the PANAS scales.," J. Pers. Soc. Psychol., vol. 54, no. 6, pp. 1063-1070, Jun. 1988, doi: 10.1037/0022-3514.54.6.1063.

[59] V. Manera et al., “"Kitchen and cooking,'a serious game for mild cognitive impairment and Alzheimer's disease: a pilot study," Front. Aging Neurosci., vol. 7, p. 24, Mar. 2015, doi: 10.3389/fnagi.2015.00024.

[60] C. E. Westerberg et al., "Concurrent impairments in sleep and memory in amnestic mild cognitive impairment,” J. Int. Neuropsychol. Soc., vol. 18, no. 3, pp. 490-500, May 2012, doi: 10.1017/S135561771200001X.

[61] D. Watson and L. A. Clark, "The PANAS-X: Manual for the positive and negative affect 
pag. 58

schedule-expanded form," 1999.

[62] S. Isaac and W. B. Michael, Handbook in research and evaluation: A collection of principles, methods, and strategies useful in the planning, design, and evaluation of studies in education and the behavioral sciences. Edits publishers, 1995.

[63] T. Anisimova, "Enhancing multi-method research methodologies for more informed decision-making," J. Adm. Gov., vol. 7, no. 1, pp. 96-104, 2012.

[64] A. Milewski-Lopez, E. Greco, F. van den Berg, L. P. McAvinue, S. McGuire, and I. H. Robertson H., "An evaluation of alertness training for older adults," Front. Aging Neurosci., vol. 6, no. 67, Apr. 2014, doi: 10.3389/fnagi.2014.00067.

[65] P. J. Silvia, Exploring the psychology of interest. oxford university Press, 2006, 10.1093/acprof:oso/9780195158557.001.0001.

[66] T. F. Van de Mortel and F. Thea, "Faking it: social desirability response bias in self-report research," Aust. J. Adv. Nursing, vol. 25, no. 4, pp. 40-48, Jun. 2008.

[67] R. E. Ready, B. R. Ott, J. Grace, and D. A. Cahn-Weiner, "Apathy and executive dysfunction in mild cognitive impairment and Alzheimer disease," Am. J. Geriatr. Psychiatry, vol. 11, no. 2, pp. 222-228, Mar. 2003, doi: 10.1097/00019442-200303000-00013. 